\title{
Quantitative characteristic of phase signal with changed pulse in the coherent $\Phi$-OTDR system
}

\author{
Zhen Zhong, ${ }^{*}$ and Xuping Zhang \\ Key Laboratory of Intelligent Optical Sensing and Manipulation, Ministry of Education, Nanjing University, Nanjing \\ 210093, China \\ *zzhong@cz-hlgd.win
}

\begin{abstract}
In the coherent $\Phi-O T D R$ system, the phase signal is retrieved based on the reference point and the observation point which are off and closer to the two sides of step of the phase change. In the experiment, the optical pulse with the changed peak power, width or shape is injected into the fiber for interrogating the change of the quantitative characteristic of the measured phase signal. When the pulse width is fixed at $200 \mathrm{~ns}$ and its peak power is adjusted from $14 \mathrm{dBm}$ to $-23 \mathrm{dBm}$, the amplitude is slightly increased from $17.3575 \mathrm{rad}$ to $17.4411 \mathrm{rad}$ as long as the Rayleigh backscattering signal can be found in the electrical signal. Changing the pulse width from $260 \mathrm{~ns}$ to $80 \mathrm{~ns}$ when the peak power is fixed at $14 \mathrm{dBm}$, the maximum amplitude and the minimum amplitude of the measured phase signal are 17.4625 rad to 17.4509 $\mathrm{rad}$, respectively. When the arbitrary shape of the optical pulse generated from the MZI structure with a changed delay fiber from $3 \mathrm{~m}$ to $6 \mathrm{~m}$, the amplitude varies from $17.4558 \mathrm{rad}$ to 17.4819 rad. For every measurement, the change of frequency is also small. And the small value of standard deviation supports the accuracy of the measurement. All the measurements show that the changed pulse nearly has no impact on the quantitative characteristic of the measured phase signal in the coherent $\Phi$-OTDR system.
\end{abstract}

Key words: Quantitative characteristic; Changed pulse; Coherent $Ф-O T D R$

\section{Introduction}

Phase optical time domain reflectometer ( $\Phi-O T D R)$ is a kind of sensing equipment which was initially used in the field of dynamic event detecting such as structure health monitoring. It achieves this function of detecting based on the sensitivity of Rayleigh backscattering light in the optical fiber. Because the laser injected into the fiber is highly coherent, Rayleigh backscattering light along the sensing fiber shows an obvious characteristic of interference which is usually in the form of the speckle-like OTDR trace [1-3]. As a matter of fact, the interference characteristic is caused by the accumulation of Rayleigh backscattering lightwaves' optical path differences while these scattered lightwaves are reflected from the scattered particles. Conversely, we can retrieve the statistical phase from these OTDR traces [4-6]. Moreover, there is a linear relationship between the phase change and the external perturbation acting on the fiber [7]. It means that once the statistical phase of $\Phi-O T D R$ is obtained, the quantitative information of the external perturbation is acquired. Therefore, a lot of researchers have studied the extraction of phase change in $\Phi-O T D R$.

In [8], the chirped pulse has been introduced to direct detection based $\Phi$-OTDR to realize the measurement of temperature and strain. In [9], the multiplexer and the circulator have been used to divide two mode lights returned from a two-mode fiber and the phase extraction was successfully executed in each mode channel. In [10], two mutually orthogonal complementary pairs of Golay sequence together with the polarization diversity has been utilized to obtain the Jones matrix. Correspondingly, the phase information was acquired from the matrix. In [6], the direct detection based $\Phi-O T D R$ with or without the interference structure has demodulated the phase of vibration by the three-port coupler. In [11], the waveform of multi-event was retrieved in $\Phi-O T D R$ based on the dual-pulse heterodyne detection.

As above has been discussed, several structures have been developed to retrieve the phase information on the fiber. Among these structures, the coherent detection system which is widely 
used in the field of optical measurement is consequently and largely applied in the dynamic measurement of $\Phi-O T D R$ due to its simple configuration and long sensing range [12-14]. In this structure, H. Cai et al. have put forward the concept of digital coherent demodulation and successfully realized the phase extraction in the $\Phi-O T D R$ system [15]. G. Tu et al. have explained the principle of the phase by the method of statistical mathematics, and the phase obtained by the experiment is highly consistent with the waveform of the applied voltage [16]. Y. Dong et al. have retrieved the fiber-stretching signal based on the principle of IQ demodulation. The linear relationship between the phase signal and the dynamic strain is revealed [17]. X. Fan et al. have demodulated the phase information successfully by Hilbert transform, and deeply studied the noise of phase [18-19]. F. Pang et al. have obtained the spatial distribution characteristic of the statistical phase in the coherent $\Phi$-OTDR system when the phase demodulation is used to resolve the fading discrimination [20].

These work have greatly promoted the research of quantitative measurement in the coherent $\Phi-O T D R$ system. However, the optical power decreases gradually as the light propagates along the optical fiber, which is an inevitable problem in the practical application [21]. To the best of our knowledge, so far, no attention has been paid to the effect of power change on the result of dynamic measurement. On the other hand, in order to avoid the impact of nonlinear effect and enhance the intensity of the Rayleigh back scattering signal, the pulse width is usually broadened [22]. If the optical power or the pulse width affects quantitative measurement, the conversion coefficient between the measured phase signal and the applied perturbation signal should be adjusted for every position of the fiber and different fixed optical pulse. At this point, the accuracy of dynamic measurement in $\Phi$-OTDR will be seriously affected by the status of optical pulse. In this article, through changing the characteristic of optical pulse injected into the test fiber, the effect of the optical pulse on the phase signal is interrogated in detail.

\section{Experimental setup and phase extraction}

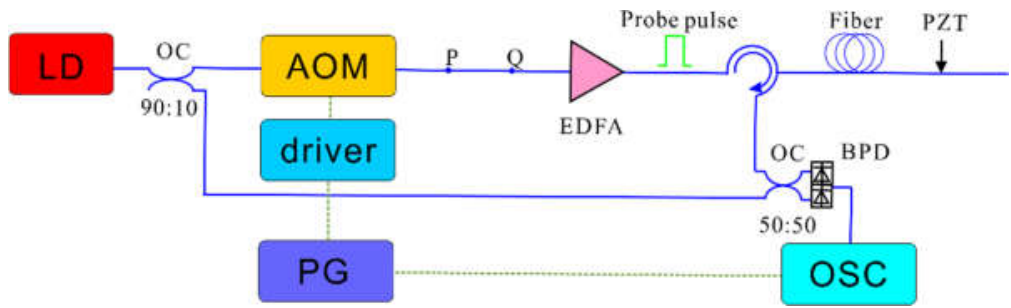

Fig. 1. Schematic of the coherent $\Phi$-OTDR system

The schematic diagram for the coherent $\Phi$-OTDR system is shown in Fig. 1. Laser LD (NKT Photonics, E15) is a highly coherent light source with the linewidth of less than $100 \mathrm{~Hz}$. The first coupler OC1 divides the light emitted from the laser into two parts by 90:10 ratio. The light of the lower branch with $10 \%$ energy is used as the local reference light. And the light of the upper branch with $90 \%$ energy is used as the probe signal light and modulated by the acoustooptic modulators, AOM with the frequency shift of 40MHz. AOM's synchronization timing with the repeat frequency of $10 \mathrm{kHz}$ is controlled by the pulse signal generator PG through the driver. Then the modulated pulse light is amplified by EDFA to the expected power and injected into the test fiber through a circulator. A piezoelectric transductor (PZT), whose driving voltage is supplied by a signal generator source, is loaded at the position of about $850 \mathrm{~m}$. The Rayleigh backscattering light returned from the optical fiber passes through the circulator into the second coupler OC2 whose splitting ratio is 50:50. At the same time, the local reference light simultaneously enters another input port of the coupler OC2. The two output ports of coupler OC2 are directly connected to the balance photodetector BPD whose electric signal is transmitted to the oscilloscope OSC synchronously triggered by PG. 
When the optical pulse with peak power of $14.03 \mathrm{dBm}$ and pulse width of $200 \mathrm{~ns}$ is injected into the test fiber for one measurement, the electric signal containing 100 OTDR traces is depicted in Fig. 2(a). Point A in Fig. 2(a) is selected as an initial reference point and corresponding phase change is acquired which is shown in Fig. 2(b). In Fig. 2(b), we can see that except for obvious noise, there is a big step change at the position of about $860 \mathrm{~m}$. For restraining the impact of the laser-frequency-drift as much as possible, the new reference point $\mathrm{B}$ and the observation point $\mathrm{C}$ which are closer to the position of step change in Fig. 2(b) are chosen. The final extracted phase signal based on points B and C is plotted in Fig. 2(c). In order to better demonstrate the characteristic of the extracted phase signal, we use a sine function with unknown parameters to fit the measured signal in Fig. 2(c). Both measured signal and fitted result are depicted in Fig. 2(d) at the same time.
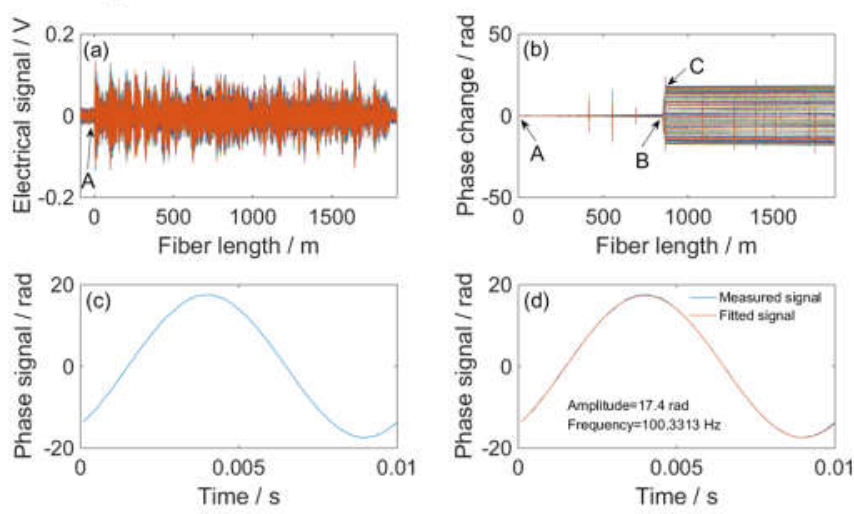

Fig. 2. Distribution of (a) electric signal and (b) phase change for one measurement (c) phase signal for vibration (d) measured phase signal and fitted phase signal

The two waveforms in Fig. 2 (d) are very consistent with each other. The fitted amplitude and the fitted frequency are $17.4 \mathrm{rad}$ and $100.3313 \mathrm{~Hz}$, respectively. For the purpose of simplification, we directly use the fitted parameters to characteristic the phase signal.

\section{Results}

In this section, the effect of amplitude, width and shape of pulse on the quantitative property will be experimentally demonstrated.

\subsection{Different amplitude of optical pulse}

The amplitude is a key parameter for the optical pulse, which is usually in the form of peak power. When the peak power of optical pulse is adjusted from $14 \mathrm{dBm}$ to $-23 \mathrm{dBm}$, each value of peak power corresponds to a distribution of electric signal displayed on the oscilloscope. Similar with Fig. 2(a), the electric signal distributes randomly along the fiber for the inhomogeneous distribution of fiber refractive index. To better demonstrate the effect of change of the peak power on the electrical signal, we do a calculation according to Eq. 1 for every measurement. For the purpose of simplification, this calculated result of Eq. 1 is called the intensity of electrical signal.

$$
I_{e}(F)=\frac{\sqrt{\sum_{i=1}^{M} \sum_{k=1}^{N}\left[I_{D}(i, k, F)\right]^{2}}}{N M}
$$

where $M$ is the maximum number of sample point along the fiber.

When the pulse width is fixed at $200 \mathrm{~ns}$, for every value of peak power, 50 times measurements are executed. And the average of the measurement results is also recorded as the intensity of electrical signa and is depicted in Fig. 3. 


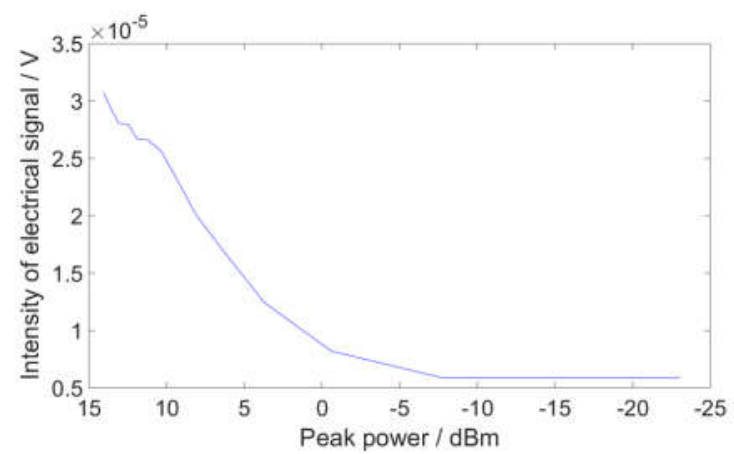

Fig. 3. Relationship between intensity of electrical signal and peak power of optical pulse

In Fig. 3, although the intensity of electrical signal doesn't vary monotonously, it decreases totally and obviously with the decay of the peak power. What should be paid more attention is that, when the peak power is adjusted from $-7.8 \mathrm{dBm}$ to $-23 \mathrm{dBm}$, the intensity of electrical signal keeps nearly unchanged, which means Rayleigh backscattering light has been submerged in the noise.

At the same time, for every measurement, the phase signal is extracted. When the peak power is adjusted from $14 \mathrm{dBm}$ to $-0.6 \mathrm{dBm}$, all the phase signals with sine waveforms are acquired. Then, with the decrease of the peak power, the phase signal in the extracted result will be no longer emerged. The amplitude and the frequency of the extracted phase signal are plotted in Fig. 4. Every displayed value in both Fig. 4(a) and Fig. 4(b) is the average of 50 times measurements for every adjusted peak power.
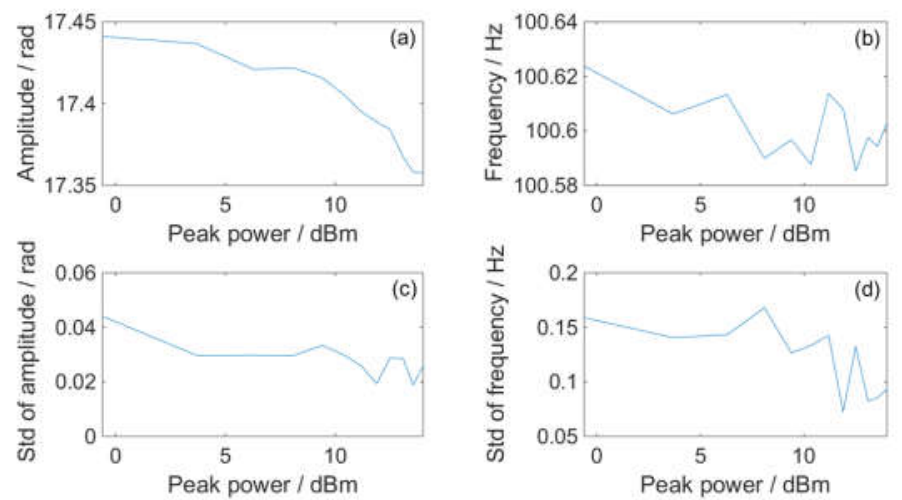

Fig. 4. Relationship between (a) amplitude (b) frequency (c) standard deviation of amplitude (d) standard deviation of frequency and peak power of optical pulse

Fig. 4 covers the range of the peak power whose intensity of electrical signal varies with the change of peak power. It means that the phase signal will be correctly extracted when Rayleigh backscattering signal in the form of the electrical signal can be detected.

From Fig. 4(a), with the decrease of peak power, the amplitude of the phase signal is slightly increased from $17.3575 \mathrm{rad}$ to $17.4411 \mathrm{rad}$. For the two values, the ratio between the difference $0.0836 \mathrm{rad}$ and the average $17.3993 \mathrm{rad}$ is $0.48 \%$ which means the slight increasement can be ignored. When the peak power is lower, the noise in the phase change trace will be increased. Correspondingly, the difficulty of choosing the reference point and the observation point is enhanced. And the amplitude of measured signal may be slightly increased due to the laser- 
frequency-drift. The standard deviation of the amplitude in Fig. 4(c) is small than 0.0441, which means that the result of every measurement is close to the true amplitude of the phase signal. The state of measured frequency is very similar with that of measured amplitude. In a word, we can deduce that the decrease of peak power does not affect the quantitative characteristic of the measured phase signal.

\subsection{Different width of optical pulse}

In order to interrogate the effect of the pulse width on the quantitative characteristic of the phase signal in the coherent $\Phi-O T D R$ system, the peak power of the optical pulse is fixed at 14 $\mathrm{dBm}$ and the pulse width is adjusted from $260 \mathrm{~ns}$ to $80 \mathrm{n}$ with a step of $30 \mathrm{~ns}$. Because the modulator in our experiment is the acousto-optic modulator, its rising edge and falling edge are relatively longer. The minimum pulse width of 80 ns makes the shape of adjusted pulse be considered as a rectangle. For every adjusted pulse width, we still execute 50 times measurements and the final measured result is the average. Through a series of calculation, All the amplitude and frequency under different pulse widths are plotted in Fig. 5(a) and Fig. 5(b). At the same time, every average has a corresponding value of standard deviation which is depicted in Fig. 5(c) and Fig. 5(d).
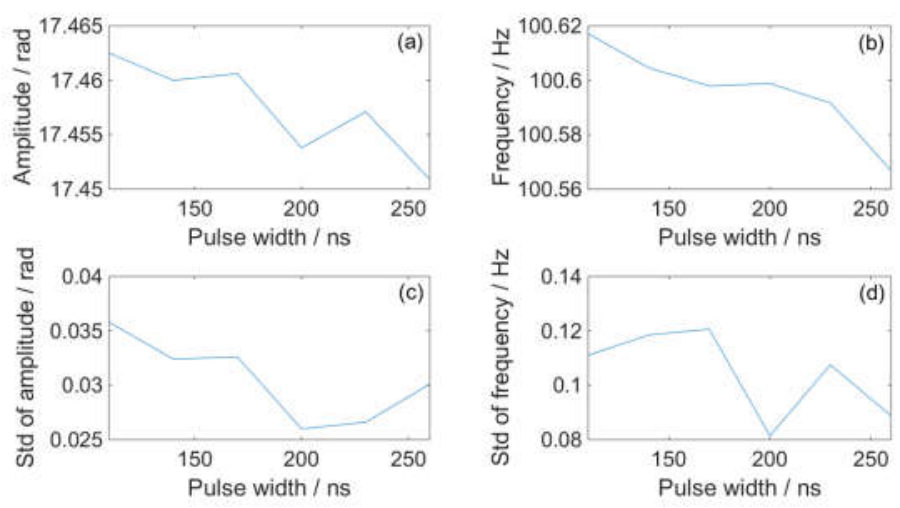

Fig. 5. Relationship between (a) amplitude (b) frequency (c) standard deviation of amplitude (d) standard deviation of frequency and pulse width

From Fig. 5(a) and Fig. 5(b), we can see that the amplitude and the frequency decrease slightly and totally with the increase of pulse width. When the pulse width is enlarged, the received energy of the Rayleigh backscattering light and the visibility are increased [22]. Correspondingly, the choose of reference point and observation point will be much easier. The closer the chosen points are to the vibration region, the smaller the influence of the measured phase signal to the laser frequency drift. Therefore, the measured amplitude is slightly and totally decreased when the pulse width is increased from 80 ns to 260 ns. However, the maximum amplitude and the minimum amplitude of the measured signal are 17.4625 rad to $17.4509 \mathrm{rad}$, respectively. For the two values, the ratio between the difference $0.0116 \mathrm{rad}$ and the average $17.4567 \mathrm{rad}$ is $0.0665 \%$ which is much less than $0.48 \%$ for Fig. 4(a). The small value $0.0665 \%$ means that the difference of amplitude in Fig. 5(a) can be ignored completely. The state of the measured frequency is very similar with that of the measured amplitude and is not described in detail. The standard deviation in Fig. 5(c) is no more than 0.358 rad which is much less than the minimum value of amplitude in Fig. 5(a). For the frequency, the ratio of the standard deviation in Fig. 5(d) to the relative measured frequency in Fig. 5(b) is less than that the standard deviation in Fig. 5(c) to the relative measured amplitude in Fig. 5(a). It means that every measurement result nearly has no measurement error and the average of amplitude and frequency in Fig. 5(a) and Fig. 5(b) can perfectly reflect the external perturbation signal. Then, 
we can reliably deduce that the change of pulse width does not change the measurement result of the external perturbation.

\subsection{Arbitrary shape of optical pulse}

In the last two small sections, the impact of the amplitude and width of the optical pulse on the extracted phase signal has been discussed. And in the experiment, the change of peak power and pulse width nearly has no impact on the characteristic (amplitude and frequency) of the measured phase signal. Therefore, we can deduce that the arbitrarily shape of optical pulse has nothing to do with the result of phase extraction. In order to demonstrate the impact of arbitrarily shape of optical pulse on the quantitative characteristic of the phase signal in the coherent $\Phi$ OTDR system, we use a short AOM based pulse to generate an arbitrarily shape of optical pulse through the MZI structure with an adjusted delay fiber. In contrast with the special shape for interrogating the effect of pulse shape on the amplitude of Rayleigh backscattering signal in [3], the shape generated in Fig. 6 has a more general representation.

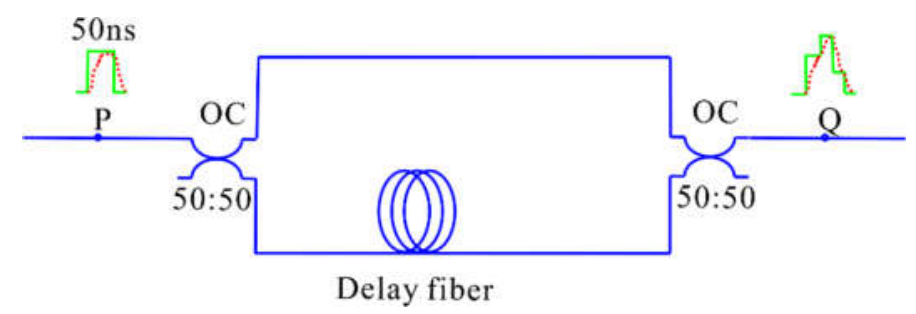

Fig. 6. Scheme of generating an arbitrary shape of optical pulse

For an AOM based optical pulse with 50ns, the front edge of the pulse has not risen to a stable status when it falls. If the width of optical pulse is larger, the rising process of optical pulse can be ignored. However, in Fig. 6, we just use the unstable status of front edge of the optical pulse to generate an arbitrary pulse. MZI structure in Fig. 6 is only used for shape generating and not for interference. In order to generate different shapes of optical pulse, the delay fiber in the MZI structure can be adjusted. The original delay fiber is $3 \mathrm{~m}$ long. Then the delay fiber is increased by the step of $1 \mathrm{~m}$. And the power loss is also increased by about 3 $\mathrm{dBm} / \mathrm{step}$. For every length of delay fiber, the quantitative result of phase extraction is depicted in Fig. 7.
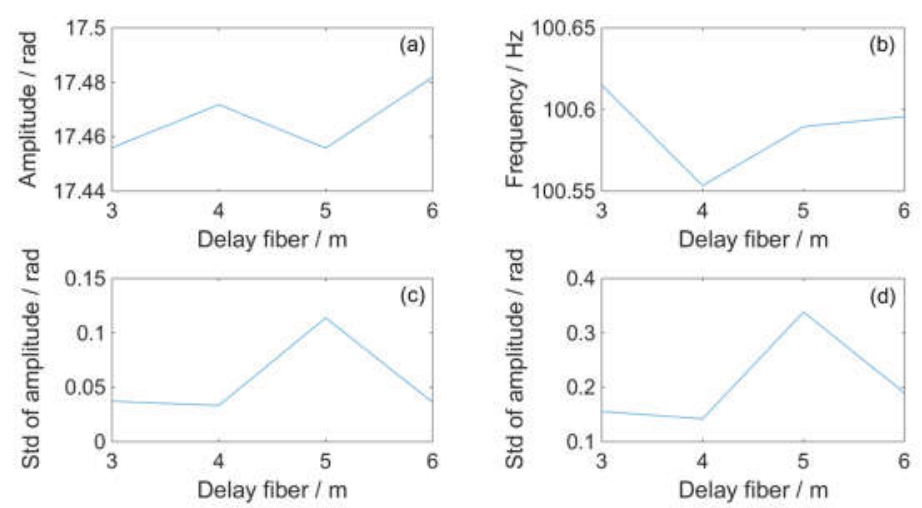

Fig. 7. Relationship between (a) amplitude (b) frequency (c) standard deviation of amplitude (d) standard deviation of frequency and length of delay fiber

From Fig. 7(a), we can see that the values of amplitude distribute in the range of [17.4558 $\mathrm{rad} 17.4819 \mathrm{rad}$ ] whose maximum difference is only $0.0261 \mathrm{rad}$ which is much less than any 
value of amplitude in Fig. 7(a). As for the measured frequency, the biggest difference in Fig. 7(b) is $0.0616 \mathrm{rad}$ which can be completely ignored when the value of frequency is compared. In Fig. 7(c) and Fig. 7(d), the distribution of standard deviation of both amplitude and frequency is very small. It also means that every measurement result has a little error. And the average of amplitude and frequency in Fig. 7(a) and Fig. 7(b) can represent the quantitative characteristic of phase signal in the coherent $\Phi-O T D R$ system. Although the pulse generated from MZI structure in Fig. 6 has a different shape due to the different length of the delay fiber, the amplitude and the frequency of the phase signal nearly have no change.

\section{Conclusion}

In the experiment, the optical pulse with changed peak power, changed pulse width or changed shape generated by a MZI structure with a 50 ns AOM pulse and a changed delay fiber is injected into the test fiber for measuring the phase signal induced by the PZT vibration. The experimental result has shown that the peak power, the pulse width and the arbitrary shape of optical pulse nearly does not make the extracted phase signal be changed. Moreover, we also find that the phase signal of external event can be correctly extracted as long as the Rayleigh backscattering signal can be detected.

\section{Funding}

This work was supported by the National Natural Science Foundation of China under Grant No. 61627816.

\section{References}

1. Y. Lu, T. Zhu, L. Chen, and X. Bao, "Distributed vibration sensor based on coherent detection of phaseOTDR,” J. Lightwave. Technol. 28, 3243 (2010).

2. J. C. Juarez, and H. F. Taylor, "Field test of a distributed fiber-optic intrusion sensor system for long perimeters," Appl. Optics. 46, 1968 (2007)

3. M. FERNÁNDEZ-RUIZ, H. Martins, J. Pastor-Graells, S. Martin-Lopez, and M. Gonzalez-Herraez, "Phasesensitive OTDR probe pulse shapes robust against modulation-instability fading," Opt. Lett. 41, 5756 (2016).

4. Z. Wang, L. Zhang, S. Wang, N. Xue, F. Peng, M. Fan, W. Sun, X. Qian, J. Rao, and Y. Rao, "Coherent $\Phi-$ OTDR based on I/Q demodulation and homodyne detection,” Opt. Express. 24, 853 (2016).

5. G. Fang, T. Xu, S. Feng, and F. Li, "Phase-sensitive optical time domain reflectometer based on phase generated carrier algorithm," J. Lightwave. Technol. 33, 2811 (2015).

6. C. Wang, Y. Shang, W. Zhao, X. Liu, C. Wang, and G. Peng, "Investigation and comparison of $\phi-O T D R$ and OTDR-interferometry via phase demodulation,” IEEE Sens. J. 18, 1501 (2018).

7. Y. Fu, N. Xue, Z. Wang, B. Zhang, J. Xiong, and Y. Rao, "Impact of I/Q amplitude imbalance on coherent Ф-OTDR," J. Lightwave. Technol. 36, 1069 (2018).

8. J. Pastor-Graells, H. Martins, A. Garcia-Ruiz, S. Martin-Lopez, and M. Gonzalez-Herraez, "Single-shot distributed temperature and strain tracking using direct detection phase-sensitive OTDR with chirped pulses," Opt. Express. 24, 13121 (2016).

9. M. Chen, A. Masoudi, F. Parmigiani, and G. Brambilla, "Distributed acoustic sensor based on a two mode fiber," Opt. Express. 26, 25399 (2018).

10. C. Dorize, and E. Awwad, "Enhancing the performance of coherent OTDR systems with polarization diversity complementary codes," Opt. Express. 26, 12878 (2018).

11. X. He, S. Xie, F. Liu, S. Cao, L. Gu, X. Zheng and M. Zhang, "Multi-event shape-retrieved distributed optical fiber acoustic sensor using dual-pulse heterodyne phase-sensitive OTDR," Opt. Lett. 42,442 (2017).

12. W. Zhang, W. Gao, L. Huang, D. Mao, B. Jiang, F. Gao, D. Yang, G. Zhang, J. Xu and J. Zhao, "Optical heterodyne micro-vibration measurement based on all-fiber acousto-optic frequency shifter" Opt. Express. 23,238105 (2015).

13. W. Choi, C. Fang-Yen, K. Badizadegan, S. Oh, N. Lue, R. Dasari and M. Feld, "Tomographic phase microscopy," Nat. Methods. 4,717 (2007).

14. F. Jiang, H. Li, Z. Zhang, Z. Hu, Y. Hu, Y. Zhang and X. Zhang, "Undersampling for fiber distributed acoustic sensing based on coherent phase-OTDR," Opt. Lett. 44,911 (2019).

15. Z. Pan, K. Liang, Q. Ye, H. Cai, R. Qu, and Z. Fang, "Phase-sensitive OTDR system based on digital coherent detection," Asia Communications \& Photonics Conference \& Exhibition. 8311, 83110S (2012).

16. G. Tu, X. Zhang, Y. Zhang, F. Zhu, L. Xia, and B. Nakarmi, "The development of an $\Phi-$ OTDR system for quantitative vibration measurement," IEEE Photonic. Tech. L. 27, 1349 (2015). 
17. Y. Dong, X. Chen, E. Liu, C. Fu, H. Zhang, and Z. Lu, "Quantitative measurement of dynamic nanostrain based on a phase-sensitive optical time domain reflectometer," Appl. Optics. 55, 7810 (2016).

18. X. Fan, G. Yang, S. Wang, Q. Liu, and Z. He, "Distributed fiber-optic vibration sensing based on phase extraction from optical reflectometry," J. Lightwave. Technol. 35, 3281 (2017).

19. G. Yang, X. Fan, S. Wang, B. Wang, Q. Liu, and Z. He, "Long-range distributed vibration sensing based on phase extraction from phase-sensitive OTDR,” IEEE Photonics. J. 8, 6802412 (2016).

20. F. Pang, M. He, H. Liu, J. Tao, T. Zhang, X. Zhang, N. Chen, and T. Wang, "A fading-discrimination method for distributed vibration sensor using coherent detection of $\varphi$-OTDR," IEEE Photonic. Tech. L. 8, 6802412 (2016).

21. H. Izumita, S. Furukawa, Y. Koyamada, and I. Sankawa, "Fading noise reduction in coherent OTDR," IEEE Photonic. Tech. L. 4, 201 (1992).

22. X. Zhong, C. Zhang, L. Li, S. Liang, H. Li, and C. Sun, "Influences of pulse on phase-sensitivity optical time domain reflectometer based distributed vibration sensor," Opt. Commun. 361, 1 (2016). 